\title{
The Design of a Real-time Monitoring System for Soil Salinization Prevention based on the Internet of Things Concept
}

\author{
Chen Guangquan ${ }^{a^{*}}$, Fu Tengfei ${ }^{b}$, Xu Xingyong ${ }^{c}$, Liu Wenquan ${ }^{d}$ \\ Key Laboratory of Marine Sedimentology and Environmental Geology, First Institute of \\ Oceanography, State Oceanic Administration, Qingdao, China \\ achenguangquan@fio.org.cn, ${ }^{\mathrm{b}}$ Futengfei@fio.org.cn, ${ }^{\mathrm{C} X u x i o n g y o n g @ f i o . o r g . c n, ~}$ \\ 'Liuwenquan@fio.org.cn
}

\begin{abstract}
Keywords: soil salinization; real-time monitoring; Internet of things; sensor
Abstract. This paper describes a system based on the concept of the Internet of Things (IoT) that provides in situ monitoring of coastal soil salinization. The system includes a central control module, as well as modules for communications, the measurement of soil parameters, and power management. The main controller is based on a CC2530 microcontroller (MCU), and a GTM900-C GSP/GPRS communication module is used to provide connectivity to the Internet. Salinization levels are obtained by measuring the electrical conductivity of the soil using a four needle probe, and sensors to measure the soil temperature and moisture content are also included. The network protocol software and client application are also designed in order to provide a full system that is capable of real-time distribution, storage, queries, and management of the measured soil salinization data.
\end{abstract}

\section{Introduction}

Saline soil is one of five major soil problems, and is widely distributed throughout the world. Saline soil is divided into the categories of inland saline soil and inshore saline soil, depending on the geographical location. The moisture and salt content of inshore saline soil fluctuates widely due to the intrusion of sea water into the shallow buried groundwater table, which increases the soil salinity upon evaporation. It is therefore desired to implement a system to automatically monitor the level of saline in the soil for intelligent management of land resources.

Saline soil monitoring is a well established research area, and specific methods of detecting the saline level include directly measuring the soil properties, analyzing remote sensing images, and electrical measurements (including the electrical conductivity measurement method, the electromagnetic measurement method, and time domain reflectometry (TDR) with intelligent microelements) ${ }^{[1-5]}$. Naturally, each method has its advantages and disadvantages. For example, the direct measurement method is simple and accurate, but is more time-consuming than other methods. Analyzing remote sensing images can be performed with a high degree of automation, but a basis of abundant data is needed. Obtaining the soil salinity through electrical measurements is widely used because it is a non-destructive test, the data is easy to obtain, and it is compatible with sequential analysis. However, the influence of other factors on the electrical properties of the soil (such as temperature and moisture content) must be considered to obtain an accurate salinity measurement.

Although various monitoring technologies are relatively mature, most of them currently only sample or monitor the soil periodically. An efficient solution that performs in situ monitoring of soil parameters and can be managed remotely is needed. The technology developed for the Internet of Things (IoT) can be used to solve the above problems. In this paper we describe a soil monitoring system that combines local terminals for in situ monitoring of soil properties and wireless capabilities for remote management. Once the data is collected by the local terminal, it is sent via a wireless network to a remote monitoring center where the measurement data is stored, processed, and released. The system provides hardware and software support for the evaluation and early warning analysis of trends in soil properties within the monitoring area, and can facilitate the effective utilization and management of coastal reserve land resources. 
This paper will first describe the architecture of the proposed system, followed by a description of the hardware modules. The software for the sensing system and remote management system will then be described, followed by concluding remarks.

\section{System architecture}

The overall structure of the system is shown in Fig. 1, and it includes the sensor terminal, a central control module composed of a microcontroller (MCU) and an EEPROM memory, and a system PC that serves as the monitoring and management system (MMS). The sensors are connected to the central control module with flexible cable, and the central control module is wirelessly connected to the MMS to implement the IoT concept. Instructions (such as starting and ending times, monitoring frequency, etc.) are issued wirelessly from the MMS to the central control module to synchronously collect the temperature, electrical conductivity, and moisture content of the soil as a function of time. The monitored data is simultaneously stored in the central control module and sent wirelessly to the MMS, where the data is distributed, stored, queried, and managed.
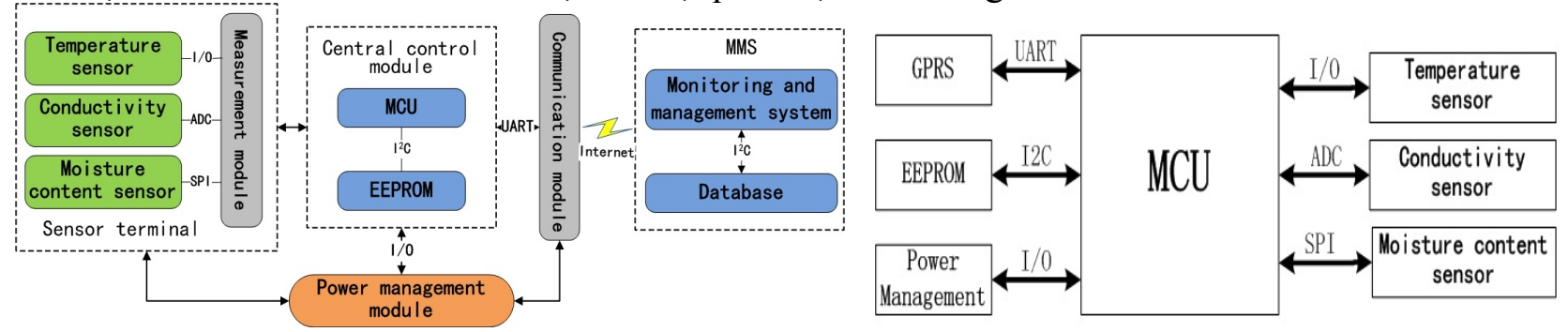

Fig. 1. System diagram of the real-time soil salinization monitoring system.

Fig. 2. Hardware diagram of the sensor system.

\section{Description of the Sensor System Hardware}

Based on the desired system functionality, an electrical measurement scheme is chosen as the soil measurement method. The system hardware is composed of four modules: the central control module, the communication module, the soil parameter measurement module, and the power management module. A hardware description of each module will be given in this section.

Central Control Module. This is the core control module of the system, and is composed of an MCU, an EEPROM memory, and peripheral circuits. The MCU is programmed with firmware that enables it to interface with the other modules for data collection and transmission. The nature of the connections between the central control module and the other modules in the system is shown in Fig. 2. A standard UART interface is used to communicate with the GPRS transceiver in the communication module, general purpose I/O ports are used to implement the power management, and an $\mathrm{I}^{2} \mathrm{C}$ port is used to communicate with the EEPROM.

The MCU is implemented using a CC2530 IC, which is well suited to this system. It is based on a standard 8051 kernel which reduces the development cycle, and has a variety of modes of operation, including a sleep mode which limits the current consumption to only $400 \mathrm{nA}$. While other blocks in the system can be power cycled using the power management module, the MCU is always powered. Setting it to operate in sleep mode between actions significantly reduces the power consumption of the system, which can extend the time needed between battery recharges or replacements.

GPRS Module. This module is used to implement the wireless information exchange between the monitoring system and the control center. The module operates using the GSM network, and similar to a standard cell phone, it can upload data and receive control commands via text messages. The GPRS module selected for this system is the GTM900-C from Huawei, which has an operating power consumption of $1 \mathrm{~W}$ (in the $900 \mathrm{MHz}$ band) to $2 \mathrm{~W}$ (in the $1800 \mathrm{MHz}$ band) and a standby current consumption of $\sim 3.5 \mathrm{~mA}$. Its operating temperature range meets the system requirements, and the 
methodology to integrate it into a system and control its operation is well documented. This results in a short and efficient development cycle.

Soil Parameter Measurement Module. The soil parameter measurement module consists of sensors to measure the temperature, moisture content, and electrical conductivity of the soil. The temperature sensor is a DS18B20 probe, which is encapsulated with the conductivity sensor module using a resin adhesive. The electrical conductivity sensor is shown in Fig. 3, and is composed of a sine-wave generator, a sensor probe, an ADC, and an MCU.

The sine-wave generator sends a $\pm 10 \mathrm{~V}, 1 \mathrm{kHz}$ sine wave to the sensor probe. The amplitude of this sine wave will depend on the penetration fluid conductivity of the soil that the probe is in contact with, and these results in the BSinX signal shown in Fig. 3. A dual path ADC is used to precisely measure the difference between the initial excitation sine wave (ASinX) and the sine wave from the sensor probe $(\mathrm{BSin} X)$, and the MCU uses this amplitude difference to calculate the conductivity of the soil.

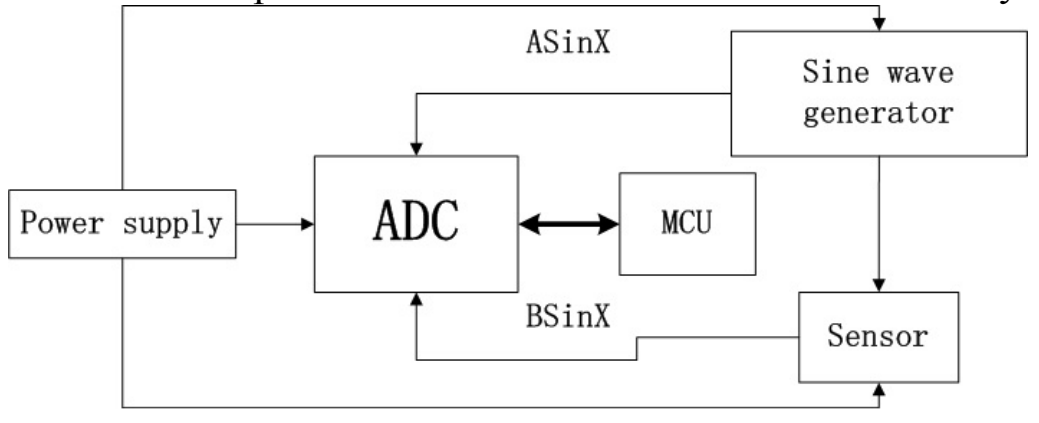

Fig. 3. Diagram of the components that comprise the conductivity sensor.

The function generator requires a very high degree of stability to avoid any measurement uncertainty due to an inaccurate excitation signal. The resolution of the ADC must also be carefully selected. A 9 bit ADC has enough resolution to satisfy the performance requirements, however, a 16 bit $\mathrm{ADC}$ is adopted to facilitate future accuracy requirements in real-time without needing any hardware modifications. The ADC communicates with the MCU through a serial SPI interface in order to minimize the required $\mathrm{MCU} \mathrm{I} / \mathrm{O}$ resources.

At present, most electrical moisture sensors are based on TDR, FDR, and standing wave theory. The essence of these measurement techniques is to calculate the moisture content of soil by measuring its dielectric constant. A typical sensor that is based on the principle of standing wave theory consists of a $100 \mathrm{MHz}$ signal source, a transmission line (that can be modeled as an LC circuit), a sensor probe, two detectors, a differential amplifier, and ancillary components. When the probe is in contact with soils of different moisture levels, the amplitude of the reflected high frequency $100 \mathrm{MHz}$ signal will vary due to the difference in the dielectric constant of the soil. The incident and reflected high frequency signals will combine to produce a standing wave pattern in the transmission line and form a wave crest and a trough at the two ends. A detector amplifies the difference in signal level between the ends of the transmission line, which provides and a one-to-one correspondence between the output voltage and the dielectric constant of the soil. The moisture content of the soil is thus measured by sampling this voltage amplitude.

Power Management Module. The function of the power management module is to minimize the power consumption of the system to extend the lifetime of the battery. It controls which modules are powered by monitoring the current mode of operation of the system. The control relationship between the power management module and the rest of the system blocks is shown in Fig. 4. 


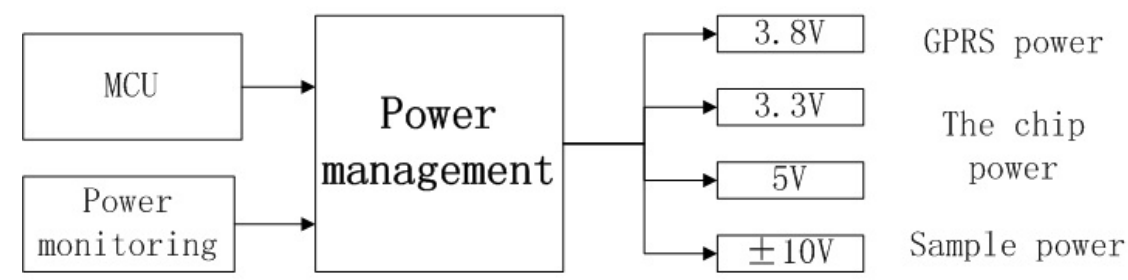

Fig. 4. Diagram of the power management system.

Each of the five blocks in the system has a unique operating voltage. The sampling circuit needs a $\pm 10 \mathrm{~V}$ power supply to operate the ADC and sine wave generator, while the GPRS module needs a 3.8 $\mathrm{V}$ power supply. The MCU, temperature sensor, and other chips need either a $3.3 \mathrm{~V}$ or a $5 \mathrm{~V}$ power supply.

A dedicated power management chip is needed to provide the required output voltages, and its operation is controlled by the MCU. The battery capacity is also monitored to provide a warning when the battery needs be recharged or replaced.

\section{Description of the System Software}

The system software design is closely related to the hardware module organization. A main program is used to control the system, and it interacts with programs designed to control the GPRS communications, the power management, the temperature measurements, the electrical conductivity measurements, and the moisture content measurements.

Main program. The primary function of the main program is to initialize the system with the correct settings and to control its operation according to the flow chart shown in Fig. 5. This program is executed upon power-up, or upon a system reset.

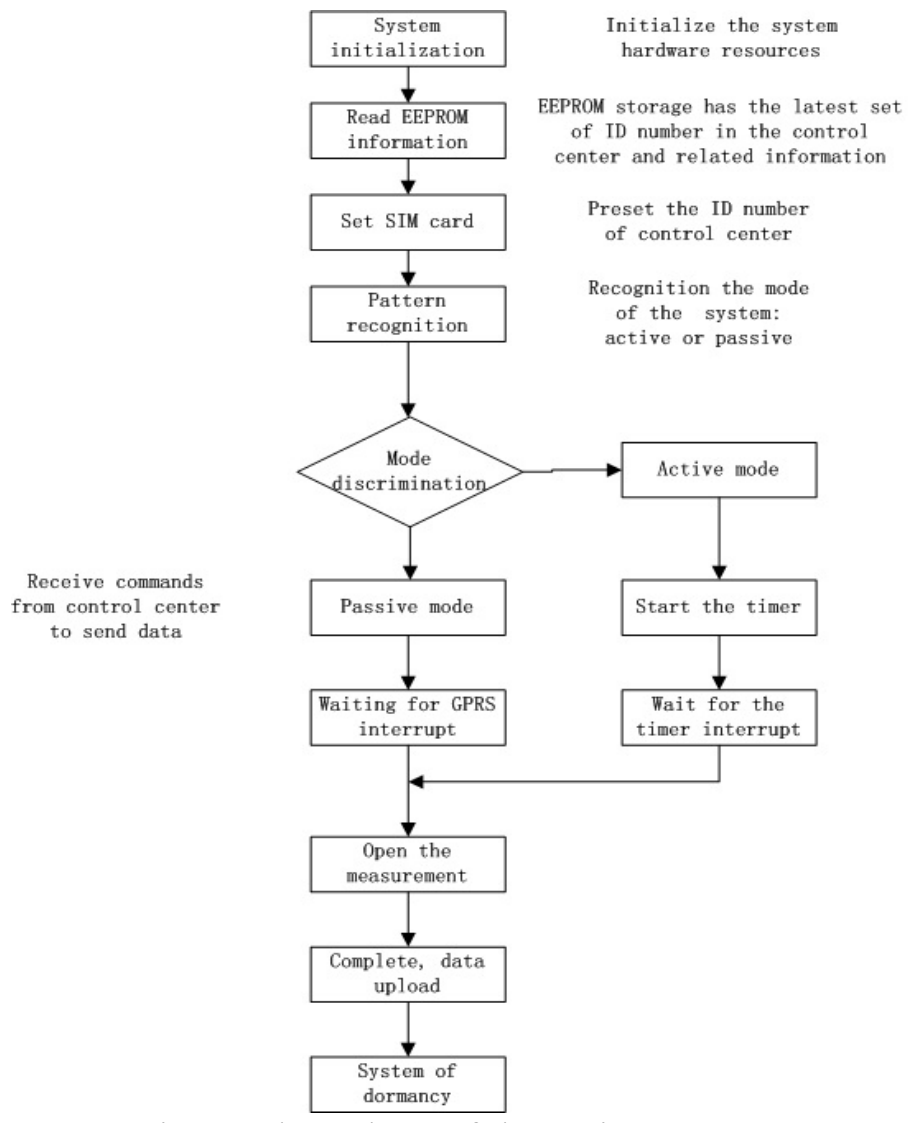

Fig. 5. Flow chart of the main program. 
GPRS Communication Program. The GPRS communication program is responsible for exchanging data between the MCU and GPRS module, as well as exchanging data between the sensor system and the remote management system.

Power Management Program. The function of this program is to control which modules and chips are powered based on the specific operations that system needs to perform at any given time.

Temperature Measurement Program. The temperature sensor is connected directly to the MCU through the I/O ports, and the measurement program is integrated into the firmware of the MCU.

Electrical Conductivity Measurement Program. This program is responsible for initiating the excitation sine wave for the conductivity measurement, and for controlling the sampling of the input and output sine waves. Since the conductivity measurement is based on measuring the amplitude changes of a sine wave, the ADC sampling frequency and channel switching time must be timed correctly to reduce the possibility of measurement error.

Moisture Content Measurement. This program controls the acquisition and processing of data from the moisture content sensor, and includes software modules to define the baud rate and implement the data transmission and processing.

\section{Soil Salinization Monitoring and Management System}

The proposed system adopts the advanced and mature model-view-controller (MVC) architecture, combined with the Java 2, enterprise edition (J2EE) platform (B/S mode), and uses a Flex $4+$ BlazeDS $4+$ Spring $3+$ MyBatis 3 multilayer development structure. The main function of the soil salinization monitoring and management system is to supply the configuration parameters to the monitoring system, and to process the received measurement data. This includes data management, analysis, and visualization, and Fig. 6 shows an image of the visualization display. This system manages the location data (city and district), the units, and the measurement data, and also provides a statistical analysis module.

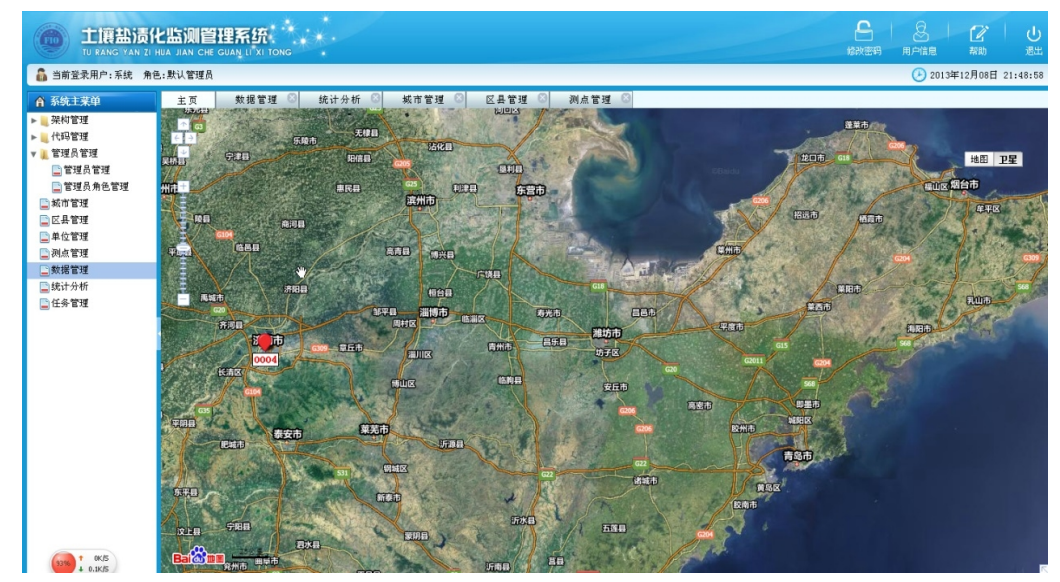

Fig. 6. An image of the system interface showing the data visualization capability.

\section{Conclusion}

In this paper, a real-time soil salinization monitoring system is developed based on the concept of the Internet of things. This solution provides in situ monitoring of inshore saline soil via a multi-parameter and automatic monitoring system with remote management capabilities. The system includes sensors to measure the temperature, moisture content, and electric conductivity of the soil. By designing and developing the central control module, communication module, soil parameter measurement module, power management module, and the client program, the system provides access to real-time soil salinization data with, storage, query, and management capabilities. These features 
provide an integrated solution that can facilitate the monitoring and prevention of soil salinization in coastal regions.

\section{Acknowledgements}

This work was financially supported by Central Public Welfare Scientific Research Institutes Foundation (GY0214G09) and Shandong Provincial Key Laboratory of Marine Ecology and Environment \& Disaster Prevention and Mitigation Foundation (201306).

\section{References}

[1] J.S. Yang. DEVELOPMENT AND PROSPECT OF THE RESEARCH ON SALT-AFFECTED SOILS IN CHINA. Acta Pedologica Sinica. 45 (2008) 837-845.

[2] G.C Topp, J.L. Davis, A.P. Annan. Electromagnetic determination of soil water content: Measurements in coaxial transmission lines. Water resources research. 16 (1980) 574-582.

[3] G.C Topp, J.L. Davis, A.P. Annan. Electromagnetic determination of soil water content using TDR: I. Applications to wetting fronts and steep gradients. Soil Science Society of America Journal. 46 (1982) 672-678.

[4] F.N. Dalton, W.N. Herkelrath, D.S. Rawlins, et al. Time-domain reflectometry: Simultaneous measurement of soil water content and electrical conductivity with a single probe. Science. 224 (1984):989-990.

[5] T.J. Heimovaara, A.G. Focke, W. Bouten, et al. Assessing temporal variations in soil water composition with time domain reflectometry. Soil Science Society of America Journal. 59 (1995) 689-698.

[6] C.F. Reece. Simple method for determining cable length resistance in time domain reflectometry systems. Soil Science Society of America Journal. 62 (1998) 314-317. 